\title{
Indistinguishability and Counting: Teaching the role of different interpretations in Quantum Mechanics
}

\author{
O.L.S. Filho ${ }^{10}$, Marcello Ferreira ${ }^{* 1 @}$ \\ ${ }^{1}$ Universidade de Brasília, Instituto de Física, Brasilia, DF, Brasil.
}

\begin{abstract}
Received on December 9, 2020. Revised on February 24, 2021. Accepted on March 23, 2021.
The theme of indistinguishability in the context of Quantum Mechanics as opposed to a distinguishability in the context of Classical Mechanics has been treated for almost a century in terms of an essential difference between the way of counting in Quantum and Classical Mechanics. Such differences are based on the adoption of the principles of Heisenberg, Duality and Complementarity. In this work, we show that it is possible to avoid such considerations and derive the same results considering only the Correspondence Principle. This is done considering that, in the classic context, the quantum discrete energy levels become continuous or almost continuous. From these results, we indicate how to develop a discussion within the scope of teaching Quantum Mechanics that involves such principles and concepts and possible comparisons, working on ideas related to the interpretation of Quantum Mechanics with regard to this phenomenon, rarely developed in traditional courses.

Keywords: Indistinguishability, Counting principles, Quantum Mechanics, Physics teaching.
\end{abstract}

\section{Introduction}

In usual courses of Quantum Mechanics, one is generally taught the three different probability weight functions, known as Boltzmann's, Bose-Einstein's and Fermi-Dirac's. It is then argued that they come from three different counting strategies, each one with its own properties and characteristics.

From Boltzmann's and Gibbs' seminal works [1], the investigations of Ehrenfest 2 and the present approaches on the subject [3], it is generally assumed that the major difference between classical and quantum counting was due to the ability to distinguish among classical particles, even if they are identical.

There are two arguments in favor of this classical distinguishability: (a) one always has trajectories in the classical domain and (b) they suffice to "follow" particles and, thus, keep track of their identities.

This framework is contrasted to the quantum one which assumes, in the context of most interpretations, some slightly different perspectives regarding the issue of trajectories within Quantum Mechanics:

1. one may have trajectories in Quantum Mechanics, but Heisenberg's Principle impedes one to follow these trajectories without error to keep track of particles - the errors grow in such a way that the particles trajectories become mixed up in an irreducible way;

2. there are no trajectories in Quantum Mechanics because, without being measured, quantum

\footnotetext{
${ }^{*}$ Correspondence email address: marcellof@unb.br
}

objects present a dual aspect, as sustained by the Duality Principle and the Complementary Principle;

Both these perspectives come from the way interpretations of Quantum Mechanics (mainly those coming from the Copenhagen Interpretation) interrelate these three fundamental principles. They are slightly different because the first can be called "epistemological", since it resides in the ability of knowing (measuring) something that do exist; while the second should be called "ontological", since it affirms that it is the physical object itself (the trajectories) that has no being [4].

At the end, these interpretations relate distinguishability with tracking capability. The fact is that it is generally assumed that classical entities, otherwise identical, can always be distinguished from each other because they can be tracked, while quantum ones should be assumed indistinguishable because we lack the hability to do so.

As is amply known, Boltzmann's weight is assumed to represent the inadequacy of Classical counting, since its use in actual calculations gives rise to Gibbs Paradox in Classical Statistical Mechanics, when it furnishes a non-extensive entropy [5]. Quantum counting, on the contrary, gives the correct extensive entropy results.

Since these results lay on quite specific perspectives related to the interpretation of the quantum formalism for this context, it would be interesting if one could present a different approach to counting that make no recourse to trajectories and the like. Such an approach would be a source for teaching some aspects regarding 
the role of interpretation within Quantum Mechanics, something not usual in courses within this theme.

In this paper, we present such an approach: we show that one can obtain the correct results for Boltzmann's weight (giving extensive entropy) assuming only that there exists a classical indistinguishability and a continuous spectrum of energy. This would serve as an example, within the subject of this paper, of the impact of assuming different interpretations for the quantum formalism.

We will show that this alternative approach not only unifies the classical and quantum ways of counting, but it also gives the correct Boltzmann's weight, removing the issue of Gibb's paradox. This latter fact can then be used to compare both approaches, revealing other possible epistemological discussions related to the interpretation of physical theory, but considered in approaches such as that of Greca \& Freire [6], which propose, in addition to the relativity of the importance of mathematical formalism, teaching in the area based on a philosophical sensitivity mediated by the following didactic binomial: balanced introduction of fundamental concepts - and strictly quantum - and consideration of interpretive issues. This perspective is timely linked to the idea that we bring about the use of Johnson-Laird's mental models as a potentially qualifying cognitive strategy of interpretation in the teaching of Quantum Mechanics.

\section{Some Trivial Results on Counting}

Let's take, as example, the following trivial situation where everyone (presumably) will agree on the way one should be counting 7 .

One has nine geometric objects as in Figure 1 . Three of them are red (R), two are yellow (Y) and four are blue (B), while the shapes are as indicated in the figure. We then ask:

- [Q1]: in how many different ways can we arrange the geometrical figures in Figure 1 with respect to color?

The terms in bold are extremely important for our interests: "arrange" tells us that we are assuming that

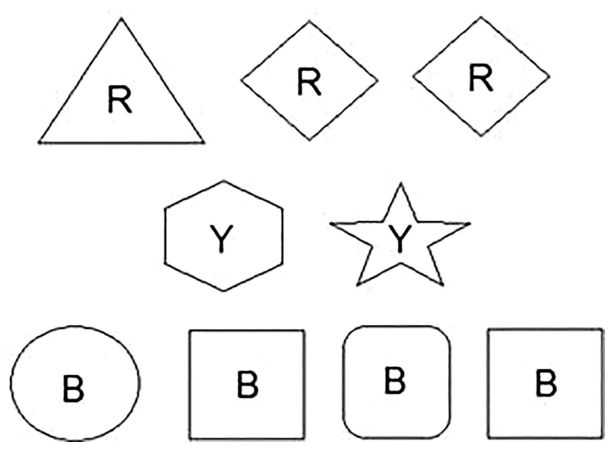

Figure 1: Nine geometrical figures with colors. Three red (R), two yellow $(\mathrm{Y})$ and four blue $(\mathrm{B})$. the order of the presentation of the colored geometrical figures matters; (b) "with respect to color" tells us the counting principle - that is, what is to be counted.

This is a trivial problem that can be found in any textbook on probability and statistics. The answer is, obviously, the weight related to a permutation with repetition:

$$
W_{Q_{1}}=\frac{9 !}{3 ! 2 ! 4 !},
$$

since, with respect to color, the first three geometrical objects are considered indistinguishable, as with the fourth and fifth yellow objects and also the last four blue objects. This is why one has a 3 !, a 2 ! and a 4 ! in the denominator of (1).

Of course, in the case of $n$ objects that can be considered indistinguishable with respect to some property, we would get simply:

$$
W_{n}=\frac{n !}{n_{1} ! n_{2} ! \cdots n_{K} !},
$$

where $K$ is the number of different values for the property, $n$ is the number of objects and $n_{i}$ is the number of indistinguishable objects within some class defined by the property used to count (e.g. color, color plus constitution, etc).

To make our point as clearly as possible, let us now change our question to:

- [Q2]: in how many different ways can we arrange the geometrical figures of Figure 1 with respect to color and shape?

The answer is again trivial and is given by:

$$
W_{Q 2}=\frac{9 !}{1 ! 2 ! 1 ! 1 ! 1 ! 2 ! 1 !}
$$

since, now, the categories of indistinguishable objects changed when we changed our property used to count. Note that the denominators also changed.

We then learn that each $n_{i}$ ! in the denominator of (2) reflects exactly one class of indistinguishable objects, given the property used to count (the property, of course, can be complex and referring to more than one characteristic of the objects, like color and shape).

The obvious conclusion is that the concept of distinguishability is always used in an operational perspective, not an ontological one. It means nothing if, in Q1, one can "see" (or track) that some colored geometrical objects have different shapes; the property used to count being color, their distinguishability can be phrased only with respect to their color - irrespective of what "they really are".

The previous argument remains unchanged if we apply our counting skills to classical or quantum worlds. Given the properties of the objects, counting proceeds without reference to such worlds. 
In the usual argument, classical particles, when having exactly the same physical properties (as mass, charge, etc), must be considered identical. However, they also should be considered distinguishable because we can put on them some mark or simply track them from their trajectories.

However, in the usual physical situation we count how energy cells can be filled by these particles. The property used to count is energy. It is not energy \& trajectory. It is, thus, irrelevant if we can or cannot track the particles. If these particles are considered identical, the only thing that can differentiate them is the energy they assume, and this is precisely what is inscribed into the $n_{i}$ ! in the denominator of 2 - there are $n_{i}$ particles assuming energy in the cell $\left[\varepsilon_{i}, \varepsilon_{i}+\Delta \varepsilon_{i}\right], n_{j}$ particles assuming energy in cell $\left[\varepsilon_{j}, \varepsilon_{j}+\Delta \varepsilon_{j}\right]$, etc.

In fact, if tracking capabilities were to be assumed within the counting principle, then there should be no $n_{i}$ ! in the denominator, since we would have to consider all particles "distinguishable", since all particles have different trajectories (or are associated to different numbers, or colors, or can be seen by us as being different), and the [sole complex] property used to count should be "particles with different trajectories and energies" this is completely analogous to the "shape and color geometrical objects" already mentioned. Thus, there would be no $n_{j}$ in the denominator. The number of ways to arrange them would then be equal to the number of ways to permute them, and counting would fail to give Boltzmann's probability density function.

\section{A Different Approach}

In agreement with the above arguments, which disregards some possible "tracking capability", we may now ask in how many different ways we can fill our energy boxes (or colored boxes) [8]. This means that, in all situations in which we have only one set of quantum objects (e.g. only photons or only electrons) presenting energies filling different energy cells, we would have to combine (not arrange) these objects into the energy cells with possible repetitions. Lets consider, again, the problem in the language of geometrical figures:

Consider three colored boxes with sub boxes, one Red (sub boxes light red and dark red, that is, degenerate by $g_{R}=2$ with respect to red color), one Yellow (sub boxes light yellow and dark yellow, $g_{Y}=2$ ), and one Blue (sub boxes light blue, medium blue and dark blue, $g_{B}=3$ ) and let us ask:

- [Q3]: in how many ways we can fill these colored boxes with $N$ geometrical figures such that we put $n_{R}$ into the Red box, $n_{Y}$ into the Yellow box and $n_{B}$ into the Blue box?

Note that, by uttering the counting principle as related to "geometrical figures", all geometrical figures become identical (no matter if they have different colors or shapes).

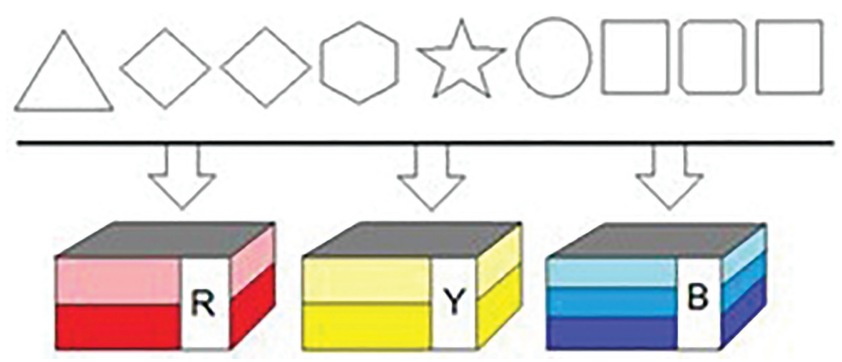

Figure 2: Ways to combine nine geometrical objects into three degenerate stages with degenerate degree numbers equal to 2 , 2 and 3 .

It is easy to see that we get simply (see Figure 2):

$W_{Q 3}=\frac{\left(n_{R}+g_{R}-1\right) !}{n_{R} !\left(g_{R}-1\right) !} \cdot \frac{\left(n_{Y}+g_{Y}-1\right) !}{n_{Y} !\left(g_{Y}-1\right) !} \cdot \frac{\left(n_{B}+g_{B}-1\right) !}{n_{B} !\left(g_{B}-1\right) !}$,

such that $N=n_{R}+n_{Y}+n_{B}$, since, fixing this last expression as a constraint, the three factors above represent independent probabilities. The result we get of the previous counting furnishes the ways we can get $n_{R}$ Red, $n_{Y}$ Yellow and $n_{B}$ Blue geometric figures (where we are counting each shade of color as "degenerate" with respect to the underlying color).

The differences are obvious if we take a look at Figure 10-2 and compare it with Figure 10-1. In Figure 10-2 the geometrical figures lost their identification labels (colors), which were now passed to the boxes, meaning that the geometrical figures are all identical (as geometrical objects [as quantum objects]) no matter how different they may be in shape [in trajectories, labels, etc.] That is, all figures [objects] are entitled to occupy any one of the degenerate shade-states [degenerate energy states] of the color-boxes [energy boxes].

This colorful example is completely analogous to the usual Quantum Mechanical counting of bosons. Again, we must stress that the fact that the geometrical figures may be "visually distinguishably" meant nothing to the outcome of our counting - they are being counted as geometrical figures [identical objects], not as geometrical figures of some shape or another [identical objects with some trajectory or another].

\section{The Usual Way of Counting}

Boltzmann's weight function is generally presented compared to the quantum weight functions. Table 1 shows the usual approach of textbooks in giving an example of the three ways of counting for two particles occupying two degenerate states.

The first counting assumes that the particles are distinguishable; thus, there are two possibilities for state $(1,1)$; the other two counting strategies assume indistinguishability, the last one assuming also Pauli's Exclusion Principle. 
Table 1: Usual counting process for Boltzmann's, BoseEinstein's and Fermi-Dirac's distributions.

\begin{tabular}{|c|c|c|c|c|c|c|}
\hline & \multicolumn{5}{|c|}{ Distributions } \\
\hline & \multicolumn{2}{|c|}{ Boltzmann } & \multicolumn{2}{|c|}{ Bose-Einstein } & \multicolumn{2}{c|}{ Fermi-Dirac } \\
\hline$(2,0)$ & $\mathrm{ab}$ & $\emptyset$ & $\mathrm{aa}$ & $\emptyset$ & $\emptyset$ & $\emptyset$ \\
\hline$(0,2)$ & $\emptyset$ & $\mathrm{ab}$ & $\emptyset$ & $\mathrm{a} a$ & $\emptyset$ & $\emptyset$ \\
\hline \multirow{2}{*}{$(1,1)$} & $\mathrm{a}$ & $\mathrm{b}$ & $\mathrm{a}$ & $\mathrm{a}$ & $\mathrm{a}$ & $\mathrm{a}$ \\
\cline { 2 - 3 } & $\mathrm{b}$ & $\mathrm{a}$ & $\mathrm{a}$ & & & \\
\hline
\end{tabular}

We note that the way of counting for Bose-Einstein and Fermi-Dirac are equivalent, but Fermi-Dirac's counting principle includes half-integral spin particles, which are governed by Pauli's Principle. Thus the first two lines of Fermi-Dirac's distribution cannot be filled, since they will assume two quantum objects in the same energy cell. These ways of counting (combinations) are qualitatively different from the way of counting that gives rise to Boltzmann's weight (arrange).

If one generalizes the results of Table 1 for $g_{i}$ states with $n_{i}$ particles, one gets the following weight functions:

$$
\begin{aligned}
W_{B}^{*}\left(\left\{n_{i}\right\}\right) & =N ! \prod_{j=1}^{K} \frac{g_{j}^{n_{j}}}{n_{j} !} \\
W_{B E}\left(\left\{n_{i}\right\}\right) & =\prod_{j=1}^{K} \frac{\left(g_{j}-1+n_{j}\right) !}{\left(g_{j}-1\right) ! n_{j} !}, \\
W_{F D}\left(\left\{n_{i}\right\}\right) & =\prod_{j=1}^{K} \frac{g_{j} !}{\left(g_{j}-n_{j}\right) ! n_{j} !}
\end{aligned}
$$

for Boltzmann's, Bose-Einstein's and Dirac's weight functions. In this case, $W_{B}^{*}$ gives an incorrect answer because of the $N$ ! factor, which makes, for instance, the entropy non-extensive in calculations for ideal gases $[9$. However, we may take another path to Boltzmann's distribution, which clarifies what we have previously said.

\section{Deducing the Correct Boltzmann's Weight}

Our first assumption was to assume the same counting principle to all three situations (Fermi-Dirac's assuming also Pauli's principle), we can find $W_{B E}$ or $W_{F D}$ as our weights (Cf. with [10]). How then can we get Boltzmann's weight from one of these (or even both)?

We thus present our second assumption: our energy states are continuous (or semi-continuous). Thus, we may take the limit $g_{j} \rightarrow \infty, \forall j$ in both $W_{B E}$ and $W_{F D}$. In this case, Stirling's approximation allows us to write $n !=n^{n} e^{-n}$ and thus:

$$
\begin{aligned}
W_{B E}\left(\left\{n_{j}\right\}\right) & =\prod_{j=1}^{K} \frac{\left(g_{j}+n_{j}\right)^{g_{j}+n_{j}}}{n_{j}^{n_{j}} g_{j}^{g_{j}}} \\
& =\prod_{j=1}^{K} \frac{g_{j}^{n_{j}}\left(1+n_{j} / g_{j}\right)^{g_{j}}}{n_{j}^{n_{j}}} ;
\end{aligned}
$$

using the fact that $(1+x / s)^{s} \rightarrow e^{x}$ if $s \rightarrow \infty$, we end with:

$$
\begin{gathered}
W_{B E}\left(\left\{n_{j}\right\}\right) \rightarrow \prod_{j=1}^{K} \frac{g_{j}^{n_{j}}}{n_{j}^{n_{j}} e^{-n_{j}}}, \\
\rightarrow \prod_{j=1}^{K} \frac{g_{j}^{n_{j}}}{n_{j} !}=W_{B}\left(\left\{n_{j}\right\}\right)
\end{gathered}
$$

where $W_{B}$ already lacks the $N$ ! term, which $W_{B}^{*}$ shows, and is devoid of problems regarding the extensive character of the entropy.

The same approach can be used for $W_{F D}$. We thus have:

$$
\begin{aligned}
W_{F D}\left(\left\{n_{j}\right\}\right)= & \prod_{j=1}^{K} \frac{g_{j}^{n_{j}}}{\left(1-n_{j} / g_{j}\right)^{g_{j}} e^{n_{j}} n_{j} !} \\
& \rightarrow \prod_{j=1}^{K} \frac{g_{j}^{n_{j}}}{n !}=W_{B}\left(\left\{n_{j}\right\}\right)
\end{aligned}
$$

Figure 3 shows the asymptotic behavior of $W_{B} / W_{F D}$ and $W_{B} / W_{B E}$ as $g$ grows with a fixed $n=200$.

Now we must ask for the interpretation of the limit $g_{j} / n_{j} \rightarrow \infty$. For the example regarding colors, this would mean that each coloured box has an infinity of possible shades, as shown in Figure 4

Clearly, this means, when it comes to quantum objects and energy boxes, that for any number of objects $n_{j}$, the amount of quantum states to be filled by them

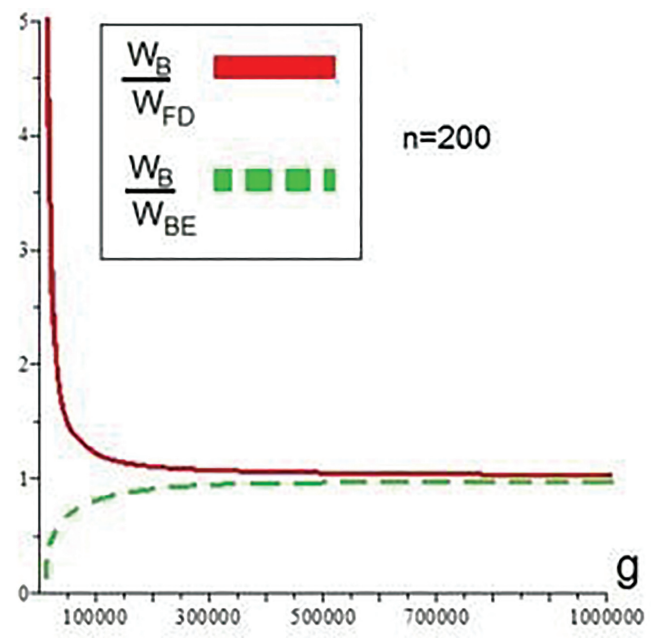

Figure 3: Asymptotic behavior of Bose-Einstein's an FermiDirac's weights as the density of states goes to infinity.

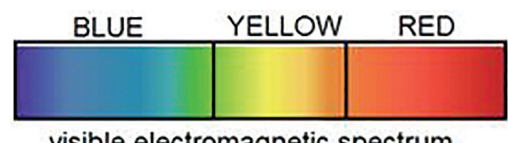

Figure 4: The same example of combination of colors, but with a continuous range of shades for each color. 
goes to a continuous or semi-continuous. This is in every sense equivalent to our combination of colors when our colored-boxes are chosen to be ranges of the electromagnetic spectrum, as in Figure 4

The conclusion is obvious enough: the only difference between Boltzmann's and Bose-Einstein's distributions (and also Fermi-Dirac's) comes from the assumption, in the derivation of the former, that energy forms a continuum. This has nothing to do with trajectories, Heisenberg's uncertainty principle, Duality principle or the Complementary principle.

In fact, making $g_{j} \rightarrow \infty$ is related to the Correspondence Principle. This shows that this principle is independent of the other three mentioned above, and can be used in alternative interpretations of Quantum Mechanics that do not take recourse to the them.

\section{Comparison Between the Two Approaches}

Now, if we count as in the first column of Table 1 for Boltzmann's weight (that is, assuming distinguishability of particles with respect to the energy), we get the physically wrong expression (because of the term $N$ !), while if we count as in the second or third columns of Table 1 for Bose-Einstein's or Fermi-Dirac's weight (that is, assuming indistinguishability with respect to the energy) and take the limit of continuous energy states we get the physically correct expression for Boltzmann's weight function.

It seems obvious that one should take the second approach, not the first. In such a case, all three weight functions come not from the assumption of some ontological indistinguishability of particles, but the assumption of a continuity of accessible states. In this framework, in a way analogous to situation Q3 already mentioned, we learn that our ability to track particles is irrelevant to the statistical result, given the counting property, which is the filling of quantum mechanical energy states.

This approach can give comprehensive grounds to the usual "large temperature limit" usually mentioned in the context of these statistical probability density. Thus, the fact that the two quantum mechanical distributions ( $\mu$ is the chemical potential):

$$
\begin{aligned}
& n_{i, B E}=\frac{g_{i}}{e^{\left(\varepsilon_{i}-\mu\right) / k_{b} T}-1}, \\
& n_{i, F D}=\frac{g_{i}}{e^{\left(\varepsilon_{i}-\mu\right) / k_{b} T}+1},
\end{aligned}
$$

tend to Boltzmann's statistical distribution

$$
n_{i, B}=e^{-\varepsilon_{i} / k_{b} T},
$$

can be interpreted as showing that the increase in temperature and low density will increase the number of accessible states, making them to tend to a quasicontinuous spectrum.
It remains for us to show how the above presented features can be approached in an actual class. We present one possibility in the next section.

\section{The Role of Mental Models in Learning: Johnson-Laird}

Johnson-Laird's mental models integrate the set of cognitive theories in which the human mind corresponds to a complex and hierarchical system: a cognitive structure capable of receiving, understanding, storing and using information. To this end, it admits that the mental models of individuals are structural analogs of the world [11, that is, internal representations of a cognitive structure which have a direct relationship with elements of the outside world.

The central role of mental models is to mediate the individual's comprehension, explanation and inference capacities about their non-cognitive analog [12]. They present themselves at different levels of complexity, ranging from those which represent simple objects, of the broad domain and acquaintance, to those that serve as scientific theories, and are characterized by nine principles [11, 12]:

1. Computability: they must be described in the form of non-dubious procedures, perfectly executable by a process or machine.

2. Finitude: deriving from the human cognition, they are associated with a vast, but limited set of possibilities for realization.

3. Constructivism: they are derivatives of essential elements that are or represent a state (or a set of states) of the outside world.

4. Economy: each configuration of the outside world is represented by only one mental model, however incipient or incomplete it may be.

5. Definiteness: they can only represent indetermination if they are circumvented by mental processing and without the exponential growth of their complexity.

6. Predictability: they must be constructed by characteristics that intersect at a point; a predicate which can apply to any element to which another predicate also applies, as long as not commutatively, to provide the distinction between natural and artificial concepts.

7. Innatism: all the conceptual primitives necessary for the construction of mental models are natural to individuals and make up their perceptual experiences.

8. A finite number of conceptual primitives: the conceptual primitives of a given semantic field are not infinite.

9. Structural identity: the structures of mental models must be identical to those of the states of the world they refer to - each element has a symbolic function and meaning. 
Being individual representations of the world state of affairs, the mental models are not necessarily accurate and consistent. Also, for this reason, they are not stationary and can be revised, complemented or even abandoned at any time [12. Thus, whenever a given mental model encounters a situation that it is unable to explain, the individual will be driven to update or, if this is not possible, to discard that model. On the other hand, competing models may be evaluated with respect to the elements just mentioned, economy being one of the most prominent and one that particularly connects to the present approach.

In the teaching field, mental models can be used to facilitate the understanding of conceptual structures, which are human constructions developed to facilitate the understanding of physical systems. Unlike mental models, conceptual models are accurate, consistent and complete representations of physical system [13].

From the teaching perspective, the teacher's role is to recover, curate, create, present and evaluate the conceptual models for their students. This set of actions should help the students to build, in their cognitive structures, mental models that are consistent with the physical systems they represent. In order to identify how these students construct such models, the teacher must seek to understand them, bearing in mind that, by their own individual nature, mental models are confusing, incomplete and unstable and that these characteristics are not indicators of an inadequate mental model [12].

The previous sections of this article, when describing the counting principles applied to the Boltzmann, Bose-Einstein and Fermi-Dirac distributions, provide the reader with competing conceptual models of the described physical systems. At the same time, when making an analogy of such systems with objects occupying spaces in colored boxes, they also try to describe a mental model under which it is possible to understand the distributions mentioned before.

In this mental model, the objects represent quantum objects, and each colored box and sub-box an energy quantum state and its degenerate states, respectively. The different combinations in which the objects can occupy the colored sub-boxes represent the different energetic degenerate states that the quantum objects could occupy.

It is essential to highlight that the mental model described, unlike the conceptual model related to the counting principles described in the previous sections, is incomplete, inconsistent and inaccurate. This idea is emphasized if it is considered that each reader forms her own mental model related to the one described to her. Again, this does not mean that they are inadequate, as they have a mutable nature and potential to evolve into models closer to physical reality. Anywise, it is possible to perceive and demonstrate that its structure is analogous to that of the described phenomena.

It is also worth noting that the article contrasts two mental models within the same theory and the same subject, thus showing how physical phenomena, not rarely, can be seen from different mental structures. This type of conflict is particularly interesting in the present case since the presented model (involving only the Principle of Correspondence, of an epistemological character) confronts an almost universally accepted model (involving the ontological character of the indistinguishability of quantum objects).

This is shown in the results obtained, which show that, whenever the number of boxes tends to infinity, the energy states can be represented as being continuous or almost continuous, which would eliminate, in the Boltzmann distribution, the error related to the entropy. The proposed mental model also allows a continuity (of an ontological nature) between the characteristics of the objects in the quantum and classical worlds (identifying them in terms of an statistical indistinguishability not ontological - hence being organized in the epistemological dimension). We believe it is unnecessary to say how much such contrast enhances the critical dimension of apprehension of the theme, in particular, and of Quantum Theory itself, in general.

Finally, it is worth noting that, in his theory, JohnsonLaird had no epistemological commitment and did not propose methods capable of guiding an instructional process through mental models. What his theory did, it should be noted, was to describe how the process of assimilation of knowledge by the cognitive structure using mental models would occur. Thereby, as we have already highlighted in another moment [14], a wellfounded and structured didactic on the theme dealt in this study should combine a descriptive theoretical perspective (cognitivist, in essence, like Laird's) with other theories or educational methodologies - therefore, of an eminently normative nature - having in mind the dual nature of such intent.

\section{Conclusion}

The obvious interpretation of the previous arguments should be that there is no inherent problem with Classical Physics (in what respects to counting). The problem resides (as it always did) in the (wrong) way many of us decided to count. In fact, the inadequacy of $W_{B}^{*}$ should have been considered from the start as an indication of problems with the chosen process of counting, not as an indication of problems with some particular domain of physics (classical or quantum). It seems that we became so eager to blame Classical Physics for each failure of some approach to a particular problem in Physics that we lost our ability to scrutinize (and, thus, understand) our own mistakes.

These arguments, together with those of previous sections, should suffice to show how classical and quantum counting can be understood as equal with respect to the notion of distinguishability, although different with respect to the number of possible degenerate 
states (where quantization reveals its importance), as expressed in the Correspondence Principle.

We believe that this would be an important contribution to explanations of the topic in our classes on Quantum Mechanics, and can give a very nice (and simple) example of the role of interpretations in Quantum Mechanics.

\section{References}

[1] J.W. Gibbs, Elementary Principles in Statistical Mechanics (Yale University Press, New Haven, 1902).

[2] P. Ehrenfest, Annalen der Physik, 36, 91 (1911).

[3] G. Baym, Lectures on Quantum Mechanics (AddisonWesley, California, 1973).

[4] S.S. Chibeni, Rev. Bras. Ens. Física 27, 181 (2005).

[5] F. Reif, Fundamentals of Statistical and thermal physics (McGraw-Hill, Singapore, 1965).

[6] https://link.springer.com/chapter/10.1007/978-94-017 -2219-3_1

[7] N. Bezerra, Análise Combinatória e Probabilidade (EditAedi, Belém, 2018).

[8] O.L.S. Filho, Quantum Mechanics: principles, new perspectives, extensions and interpretation (Nova Science, New York, 2016).

[9] https://link.springer.com/chapter/10.1007/978-94-017 -2219-3_1

[10] A. Bach, Indistinguishable Classical Particles (Springer, Berlin, 1997).

[11] P. Johnson-Laird, Mental models (Harvard University Press, Cambridge, 1983).

[12] M.A. Moreira, Investigações em Ensino de Ciências 1, 193 (1996).

[13] D.A. Norman, in: Mental models, edited by D. Gentner and A.L. Stevens (Lawrence Erlbaum Associates, Hillsdale, 1983).

[14] O.L.S. Filho and M. Ferreira, Revista do Professor de Física 2, 104 (2018). 